\title{
On Heavy-tailed Crack Distribution for Loss Severity Modeling
}

\author{
Taehan Bae ${ }^{1} \&$ Jingjiao Chen ${ }^{1}$ \\ ${ }^{1}$ Mathematics and Statistics, University of Regina, Regina, Canada \\ Correspondence: Taehan Bae, Mathematics and Statistics, University of Regina, Regina, Saskatchewan, S4S 0A2, \\ Canada. Tel: 1-306-585-4353. E-mail: taehan.bae@uregina.ca
}

Received: September 11, 2017 Accepted: September 25, 2017 Online Published: October 4, 2017

doi:10.5539/ijsp.v6n6p92

URL: https://doi.org/10.5539/ijsp.v6n6p92

\begin{abstract}
Heavy-tailedness and right-skewness are two typical features of loss data resulting from catastrophic events such as storms or earthquakes. In this paper we study the tail properties of the generalized crack distribution which has recently been introduced as an extension of the Birnbaum-Saunders distribution and the three-parameter Gaussian crack distribution. The theoretical tail relationships between the auxiliary (or baseline) distribution and the resulting generalized crack distribution are studied relying on the classical theories of extreme values and regular variation. A few concrete examples of heavy-tailed crack distribution are constructed and used for model fitting exercises on both simulated and real catastrophic loss data sets. The fitting results show that the heavy-tailed crack distribution with an appropriate choice of baseline density function outperforms some other commonly used parametric models.
\end{abstract}

Keywords: Birnbaum-Saunders distribution, generalized Birnbaum-Saunders distribution, three-parameter crack distribution, extreme value theory, regular variation

\section{Introduction}

The Birnbaum-Saunders (BS) distribution (Birnbaum \& Saunders, 1969a \& 1969b) is a two-parameter lifetime distribution constructed to model the reliability of materials or products. Due to its mathematical tractability and ability to fit positively skewed data, the BS distribution has also been used for many applications beyond the reliability analysis (Leiva, 2015). The BS distribution has recently been extended to a more general class of distributions, the so-called generalized Birnbaum-Saunders (GBS) distribution class (Diaz-Garcia \& Leiva, 2005). The GBS distribution class is built using the density functions of elliptically symmetric distributions in place of the standard normal density on which the original BS distribution relies. Thanks to its increased flexibility, the GBS distribution class has admitted a wider variety of applications including reliability theory, lifetime analysis, environmental studies and medical science; see Diaz-Garcia \& Leiva (2005), Sanhueza et al. (2008a,b) \& Leiva et al. (2008a,b) for references. More recently, Ferreira et al. (2012) discuss the tail behavior of GBS distribution in the context of the extreme value theory (e.g., Bingham et al., 1987 \& Embrechts et al., 1997). The authors show that the tail thickness of GBS distribution is essentially determined by that of the auxiliary (or baseline) distribution and thus, the GBS distribution with a heavy-tailed baseline density can be effectively used for modeling extreme data such as catastrophic losses. The interested reader is also referred to Gomes et al. (2012) \& Ferreira (2013) for some applications of extreme value BS models.

Yet another interesting extension of Birnbaum-Saunders distribution is the three-parameter (Gaussian) crack distribution (Volodin \& Dzhungurova, 2000) which is a two-point mixture of inverse Gaussian distribution and length-biased inverse Gaussian distribution. In particular, the crack distribution with the weight parameter $p=1 / 2$ reduces to the classical twoparameter BS distribution. By construction, the three-parameter crack distribution obtains a greater flexibility in fitting various asymmetric data than the BS distribution does. However, the thin-tailedness of the baseline (normal) distribution passes on to the three-parameter crack distribution, which imposes restrictions on the crack distribution for modeling heavy-tailed data. The unified approach by Leiva et al. (2010) and the generalized crack (GCR) distribution studied independently by Bae \& Volodin (2014) result in a rich class of distribution which allows for both heavy-tailedness and flexibility in fitting various distributional shapes.

This paper has three main objectives. First of all, we discuss the tail properties of GCR distribution in the context of extreme value theory. The second goal is to introduce some concrete heavy-tailed (Student's $t$-, Laplace- and Generalized Gaussian-) crack distributions for practical applications. Last but not least, by fitting real loss data sets from natural disasters, we demonstrate the applicability of these heavy-tailed crack distributions for loss severity modeling.

The rest of the paper is organized as follows. Section 2 gives a brief review of the Birnbaum-Saunders, the three-parameter crack and the generalized crack distributions. In Section 3, the tail properties of generalized crack distribution are discussed with a few concrete examples of heavy-tailed crack distributions. The model fitting results based on both simulated and real catastrophic data sets are presented in Section 4. Finally some concluding remarks are made in Section 5. Proofs 
are relegated to the appendix.

\section{Preliminaries}

In this section, we give a brief overview of the Birnbaum-Saunders distribution, the three-parameter crack distribution and the generalized crack distribution.

\subsection{The Birnbaum-Saunders Distribution}

Birnbaum \& Saunders (1969a, 1969b) discuss the failure time distribution of a material based on a fatigue process where the cumulative fatigue (or stress) in excess of a critical threshold causes the material to fail due to crack. Note that there an intimate relationship between the BS distribution and the Inverse Gaussian distribution; the Birnbaum-Saunders distribution is the mixture of an inverse Gaussian distribution and length-biased inverse Gaussian distribution with equal weights (Desmond, 1986)). Formally, the cumulative distribution function (cdf) and the probability density function (pdf), respectively, of the (two-parameter) BS distribution are as follows:

$$
\begin{aligned}
& f_{\mathrm{BS}}(t)=1-\Phi\left(\frac{1}{\alpha}\left(\sqrt{\frac{\beta}{t}}-\sqrt{\frac{t}{\beta}}\right)\right), \\
& f_{\mathrm{BS}}(t)=\frac{1}{2} f_{\mathrm{IG}}(t)+\frac{1}{2} f_{\mathrm{LB}-\mathrm{IG}}(t), \quad t>0,
\end{aligned}
$$

where

$$
\begin{aligned}
f_{\mathrm{IG}}(t) & =\frac{\sqrt{\beta}}{\alpha} t^{-3 / 2} \phi\left(\frac{1}{\alpha}\left(\sqrt{\frac{\beta}{t}}-\sqrt{\frac{t}{\beta}}\right)\right), \\
f_{\mathrm{LB}-\mathrm{IG}}(t) & =\frac{1}{\alpha \sqrt{\beta}} t^{-1 / 2} \phi\left(\frac{1}{\alpha}\left(\sqrt{\frac{\beta}{t}}-\sqrt{\frac{t}{\beta}}\right)\right),
\end{aligned}
$$

and $\alpha>0$ and $\beta>0$ are the shape and scale parameters, respectively. Here $\Phi(\cdot)$ and $\phi(\cdot)$ denote the cdf and pdf of the standard normal distribution, respectively, and $f_{\mathrm{IG}}(t)$ and $f_{\mathrm{LB}-\mathrm{IG}}(t)$ denote the pdfs of inverse Gaussian (IG) distribution and the length-biased inverse Gaussian (LB-IG) distribution, i.e., $f_{\mathrm{LB}-\mathrm{IG}}(t)=t f_{\mathrm{IG}}(t) / \beta$, respectively. See Patil (2002) for more details on length-biased distribution.

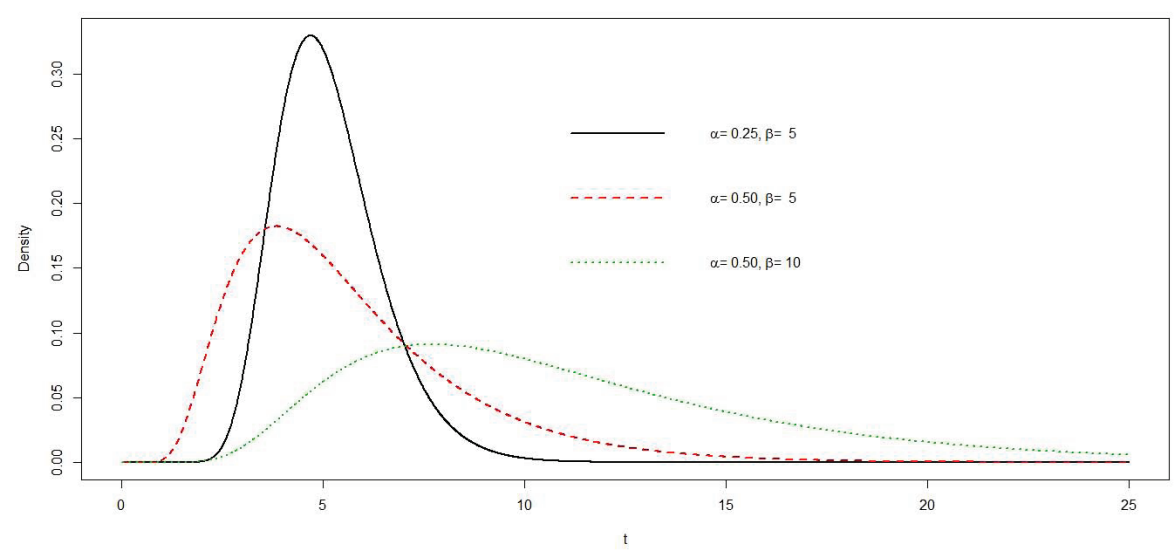

Figure 1. Density functions of BS distributions.

Figure 1 plots density curves of the Birnbaum-Saunders distribution with a few prescribed values of $\alpha$ and $\beta$. One can see from the figure that the (positive) skewness of the distribution become evident as the shape parameter gets large.

\subsection{The Three-parameter Crack Distribution}

Due to their flexibility to provide adjustments for local variations in datam, finite mixture models have been widely used in various fields such as economics, engineering and biological science. As an extension of Birnbaum-Saunders distribution, Volodin and Dzhungurova (2000) introduce the three-parameter (Gaussian) crack (lifetime) distribution which is defined as the two-point mixture of the IG distribution and LB-IG distribution with the mixing weight parameter $p \in[0,1]$. Clearly the right tail of LB-IG distribution is thicker than that of IG distribution and the mixture weight parameter $p$ 
determines the level of the tail thickness of crack distribution. Formally, the pdf of the crack (CR) distribution is given as

$$
\begin{aligned}
f_{\mathrm{CR}}(t) & =p f_{\mathrm{IG}}(t)+(1-p) f_{\mathrm{LB}-\mathrm{IG}}(t) \\
& =\left(p \frac{\sqrt{\beta}}{\alpha} t^{-3 / 2}+(1-p) \frac{1}{\alpha \sqrt{\beta}} t^{-1 / 2}\right) \phi\left(\frac{1}{\alpha}\left(\sqrt{\frac{\beta}{t}}-\sqrt{\frac{t}{\beta}}\right)\right), t>0,
\end{aligned}
$$

where $\alpha>0$ and $\beta>0$ are the shape and scale parameters, respectively, and $0 \leq p \leq 1$ is the mixture weight parameter.

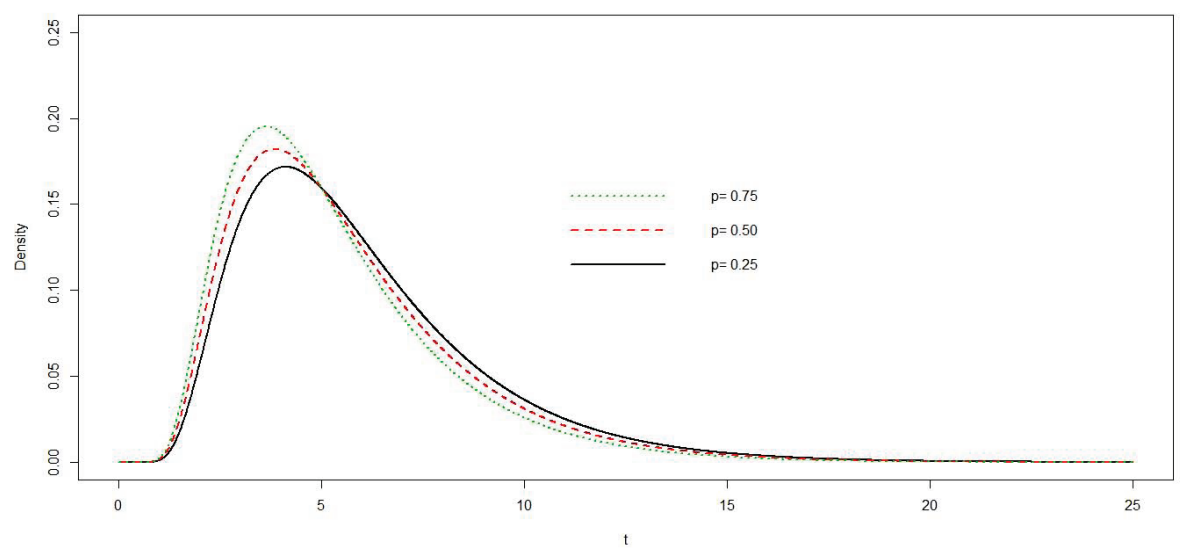

Figure 2. Density functions of crack distributions with $\alpha=0.50, \beta=5$.

Figure 2 illustrates the density curves of the Gaussian crack distribution with $\alpha=0.50, \beta=5$ and $p \in\{0.25,0.50,0.75\}$. Even though the right tail of the crack distribution becomes thicker as $p$ gets smaller, the tail resembles that of an exponential distribution. This can be seen from the fact that the limit of the hazard function converges to a constant larger than zero;

$$
\lim _{t \rightarrow \infty} \frac{f_{\mathrm{CR}}(t)}{1-F_{\mathrm{CR}}(t)}=\lim _{t \rightarrow \infty} \frac{f_{\mathrm{CR}}^{\prime}(t)}{-f_{\mathrm{CR}}(t)}=\frac{1}{2 \beta \alpha^{2}}>0 .
$$

Further details on the tail properties of crack distribution are given in Example 3.

\subsection{The Generalized Crack Distribution}

Leiva et al. (2010) discuss a unified mixture modeling approach based on inverse Gaussian distribution. Independently, Bae and Volodin (2014) study a generalization of the Gaussian crack distribution by replacing the standard normal density with an arbitrary symmetric density function. Here we present the definition of the generalized crack distribution, its theoretical moments and a random number generation method for the sake of readers' convenience.

\subsubsection{Definition}

Specifically, a random variable $T$ follows a GCR distribution, denoted by $T \sim \operatorname{GCR}(\alpha, \beta, p ; g)$, if and only if its pdf is as follows:

$$
f_{\mathrm{GCR}}^{g}(t ; \alpha, \beta, p)=p f_{\mathrm{IS}}^{g}(t ; \alpha, \beta)+(1-p) f_{\mathrm{LB}-\mathrm{IS}}^{g}(t ; \alpha, \beta), \quad t>0,
$$

where

$$
\begin{aligned}
f_{\mathrm{IS}}^{g}(t ; \alpha, \beta) & =\frac{\sqrt{\beta}}{\alpha} t^{-3 / 2} g\left(\frac{1}{\alpha}\left(\sqrt{\frac{\beta}{t}}-\sqrt{\frac{t}{\beta}}\right)\right), \\
f_{\mathrm{LB}-\mathrm{IS}}^{g}(t ; \alpha, \beta) & =\frac{1}{\alpha \sqrt{\beta}} t^{-1 / 2} g\left(\frac{1}{\alpha}\left(\sqrt{\frac{\beta}{t}}-\sqrt{\frac{t}{\beta}}\right)\right),
\end{aligned}
$$

where $\alpha>0, \beta>0,0 \leq p \leq 1$. The density function $g(\cdot)$ is symmetric around zero and referred as auxiliary (or baseline) density function. Here $f_{\mathrm{IS}}^{g}$ is called the pdf of the inverse symmetric (IS) distribution with baseline density function $g(\cdot)$. Similarly, $f_{\mathrm{LB}-\mathrm{IS}}^{g}$ is referred to as the pdf of the length-biased inverse symmetric (LB-IS) distribution with baseline density function $g(\cdot)$. 
In the following, we show that both $f_{\mathrm{IS}}^{g}$ and $f_{\mathrm{LB}-\mathrm{IS}}^{g}$ are valid density functions: Obviously, $f_{\mathrm{IS}}^{g}(t) \geq 0, f_{\mathrm{LB}-\mathrm{IS}}^{g}(t) \geq 0$ for any $t>0$. Next, we have

$$
\begin{aligned}
\int_{0}^{\infty} f_{\mathrm{IS}}^{g}(t) d t & =\int_{0}^{\infty} \frac{\sqrt{\beta}}{\alpha} t^{-\frac{3}{2}} g(s) d t \\
& =\int_{-\infty}^{\infty}\left(1+\frac{s}{\sqrt{s^{2}+4 / \alpha^{2}}}\right) g(s) d s \\
& =\int_{-\infty}^{\infty} g(s) d s+\int_{-\infty}^{\infty} \frac{s}{\sqrt{s^{2}+4 / \alpha^{2}}} g(s) d s \\
& =1+0=1,
\end{aligned}
$$

where $s=\alpha^{-1}(\sqrt{\beta / t}-\sqrt{t / \beta})$. Thus, $f_{\mathrm{IS}}^{g}(t)$ is a valid pdf. Since

$$
\frac{1}{2} \int_{0}^{\infty}\left[f_{\mathrm{IS}}^{g}(t)+f_{\mathrm{LB}-\mathrm{IS}}^{g}(t)\right] d t=\lim _{t \rightarrow \infty}\left[1-G\left(\frac{1}{\alpha}\left(\sqrt{\frac{\beta}{t}}-\sqrt{\frac{t}{\beta}}\right)\right)\right]=1,
$$

where $G(x)=\int_{-\infty}^{x} g(s) d s$. Thus, $\int_{0}^{\infty} f_{\mathrm{LB}-\mathrm{IS}}^{g}(t) d t=1$ as desired.

One can easily show that the cdf of $T \sim \mathrm{GCR}(\alpha, \beta, p ; g)$ can be expressed as follows:

$$
F_{\mathrm{GCR}}^{g}(t ; \alpha, \beta, p)=\bar{G}(b(t))+(2 p-1) \int_{\sqrt{b^{2}(t)+4 / \alpha^{2}}}^{\infty} g\left(\sqrt{x^{2}-\frac{4}{\alpha^{2}}}\right) d x,
$$

where $b(t)=\alpha^{-1}(\sqrt{\beta / t}-\sqrt{t / \beta}), \bar{G}(\cdot)=1-G(\cdot)$, and $G(\cdot)$ is the cdf of the baseline density function $g(\cdot)$. From (1), one can see that the GCR family belongs to a scale distribution family; i.e., if $T \sim \operatorname{GCR}(\alpha, \beta, p ; g)$ then $c T \sim \operatorname{GCR}(\alpha, c \beta, p ; g)$ for a positive constant $c$.

As a special case with $p=1 / 2$, the cdf of the generalized Birnbaum-Saunders distribution (Díaz-García and LeivaSánchez, 2005) with baseline density function $g(\cdot)$ is given as

$$
F_{\mathrm{GCR}}^{g}\left(t ; \alpha, \beta, \frac{1}{2}\right)=\bar{G}\left(\frac{1}{\alpha}\left(\sqrt{\frac{\beta}{t}}-\sqrt{\frac{t}{\beta}}\right)\right) .
$$

\subsubsection{Moments}

Bae and Volodin (2014) derive the expressions for the moments of GCR distributions. Here we present the results without proofs. Specifically, let a random variable $S$ have the IS distribution with baseline density function $g$, denoted by $S \sim$ IS $(\alpha, \beta ; g)$. Then, the $n$th moment of $S$ is

$$
\mathrm{E}\left[S^{n}\right]=\left(\frac{\alpha^{2} \beta}{4}\right)^{n} \sum_{k=0}^{n-1} \sum_{h=0}^{n-k-1}\left(\begin{array}{c}
2 n-1 \\
2 k
\end{array}\right)\left(\begin{array}{c}
n-k-1 \\
h
\end{array}\right)\left(\frac{4}{\alpha^{2}}\right)^{n-k-h} \mu_{2(k+h)}^{\prime},
$$

where $\mu_{(j)}^{\prime}:=\int_{-\infty}^{\infty} x^{j)} g(x) d x$. Using the relationship between the IS distribution and the LB-IS distribution, $f_{\text {LB-IS }}(t)=$ $t f_{\mathrm{IS}}(t) / \beta$, the $n$th moment of LB-IS distribution with the baseline density function $g(\cdot)$ can be easily calculated out based on the $n$th moment of IS distribution with the same baseline density function $g(\cdot)$. Suppose a random variable $U$ follows the LB-IS distribution, denoted by $U \sim \operatorname{LB}-\mathrm{IS}(\alpha, \beta ; g)$. Then, the $n$th moment of $U$ is

$$
\mathrm{E}\left[U^{n}\right]=\left(\frac{\alpha^{2} \beta}{4}\right)^{n} \sum_{k=0}^{n} \sum_{h=0}^{n-k}\left(\begin{array}{c}
2 n+1 \\
2 k
\end{array}\right)\left(\begin{array}{c}
n-k \\
h
\end{array}\right)\left(\frac{4}{\alpha^{2}}\right)^{n-k-h} \mu_{2(k+h)}^{\prime} .
$$

Since the $\operatorname{GCR}(\alpha, \beta, p ; g)$ distribution is a combination of $\operatorname{IS}(\alpha, \beta ; g)$ distribution and LB-IS $(\alpha, \beta ; g)$ distribution, the $n$th moment of GCR distribution for a random variable $T \sim \operatorname{GCR}(\alpha, \beta, p ; g)$ is then

$$
\begin{aligned}
\mathrm{E}\left[T^{n}\right]= & \left(\frac{\alpha^{2} \beta}{4}\right)^{n}\left\{\sum _ { k = 0 } ^ { n - 1 } \sum _ { h = 0 } ^ { n - k - 1 } ( \frac { 4 } { \alpha ^ { 2 } } ) ^ { n - k - h } \left[p\left(\begin{array}{c}
2 n-1 \\
2 k
\end{array}\right)\left(\begin{array}{c}
n-k-1 \\
h
\end{array}\right)\right.\right. \\
& \left.\left.+(1-p)\left(\begin{array}{c}
2 n+1 \\
2 k
\end{array}\right)\left(\begin{array}{c}
n-k \\
h
\end{array}\right)\right] \mu_{2(k+h)}^{\prime}+(1-p) 4^{n} \mu_{2 n}^{\prime}\right\} .
\end{aligned}
$$


In particular the first four moments of GCR distribution are:

$$
\begin{aligned}
\mathrm{E}[T] & =\beta\left[1+(1-p) \alpha^{2}\right] \\
\mathrm{E}\left[T^{2}\right] & =\beta^{2}\left[1+(3-2 p) \alpha^{2}+(1-p) \alpha^{4} \mu_{4}^{\prime}\right] ; \\
\mathrm{E}\left[T^{3}\right] & =\beta^{3}\left[1+3(2-p) \alpha^{2}+(5-4 p) \alpha^{4} \mu_{4}^{\prime}+(1-p) \alpha^{6} \mu_{6}^{\prime}\right] \\
\mathrm{E}\left[T^{4}\right] & =\beta^{4}\left[1+2(5-2 p) \alpha^{2}+5(3-2 p) \alpha^{4} \mu_{4}^{\prime}+(7-6 p) \alpha^{6} \mu_{6}^{\prime}+(1-p) \alpha^{8} \mu_{8}^{\prime}\right],
\end{aligned}
$$

Provided that $\mathrm{E}\left[T^{n}\right]$ exists for $n=1,2,3,4$ and letting $q=1-p$, the variance $\left(\sigma^{2}\right)$, skewness $(\gamma)$ and kurtosis $(\kappa)$ are, respectively, given by

$$
\begin{aligned}
& \sigma^{2}= \alpha^{2} \beta^{2}\left[1-\alpha^{2} q^{2}+\alpha^{2} q \mu_{4}^{\prime}\right] ; \\
& \gamma= \frac{\alpha\left[q\left(2 \alpha^{2} q^{2}-3\right)+\left(1+q-3 \alpha^{2} q^{2} \mu_{4}^{\prime}+\alpha^{2} q \mu_{6}^{\prime}\right)\right]}{\sqrt{\left(1-\alpha^{2} q^{2}+\alpha^{2} q \mu_{4}^{\prime}\right)^{3}}} ; \\
& \kappa=\frac{3 \alpha^{2} q^{2}\left(2-\alpha^{2} q^{2}\right)+\left[1-4 \alpha^{2} q(1+q)+6 \alpha^{4} q^{3}\right] \mu_{4}^{\prime}}{\left[1-\alpha^{2} q^{2}+\alpha^{2} q \mu_{4}^{\prime}\right]^{2}} \\
&+\frac{\left(\alpha^{2}+2 \alpha^{2} q-4 \alpha^{4} q^{2}\right) \mu_{6}^{\prime}+\alpha^{4} q \mu_{8}^{\prime}}{\left[1-\alpha^{2} q^{2}+\alpha^{2} q \mu_{4}^{\prime}\right]^{2}} .
\end{aligned}
$$

Figures 3 and 4 plot the skewness and kurtosis of Gaussian GCR distribution, respectively, as functions of $\alpha$ and $q$.
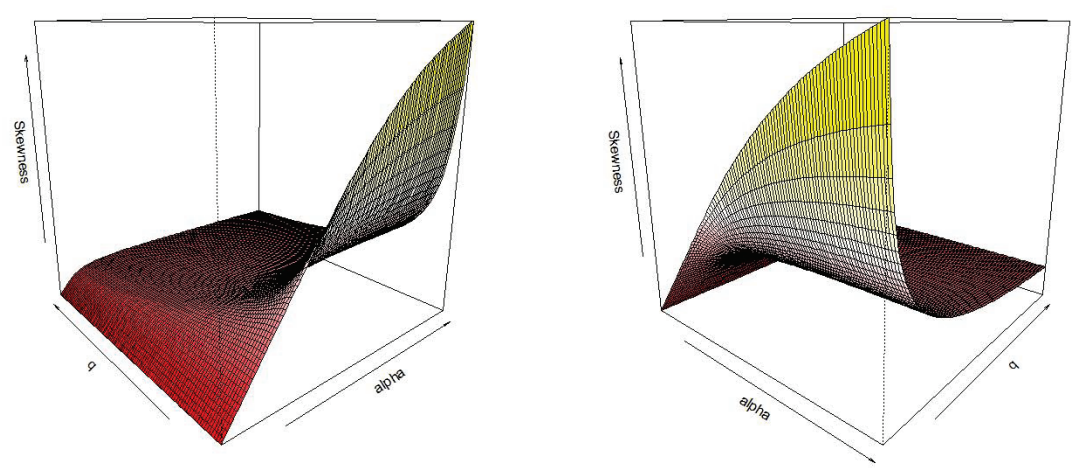

Figure 3. Skewness of GCR distribution.

\subsubsection{Generation of GCR Random Numbers}

A reliable acceptance-rejection method for GCR random number generation is discussed in Bae and Volodin (2014). The method is used for our simulation studies in Section 4.1 and, for readers' convenience, we give the algorithm to generate a random variable $T \sim \operatorname{GCR}(\alpha, \beta, p ; g)$ in the following:

Step 1. Generate a random variable $X$ from the baseline distribution $G$.

Step 2. Suppose $Y=\frac{\beta}{4}\left(-\alpha X+\sqrt{\alpha^{2} X^{2}+4}\right)^{2}$. Then $Y \sim \operatorname{GCR}(\alpha, \beta, 1 / 2 ; g)$.

Step 3. Generate a Uniform $[0,1]$ random variable $V$ independent from $X$.

Step 4. If

$$
V \leq \frac{f_{\mathrm{GCR}}^{g}(Y ; \alpha, \beta, p)}{c f_{\mathrm{GCR}}^{g}(Y ; \alpha, \beta, 1 / 2)}
$$

then accept $Y$ and let $T=Y$. Otherwise, reject $Y$ and go back to step 1 . 

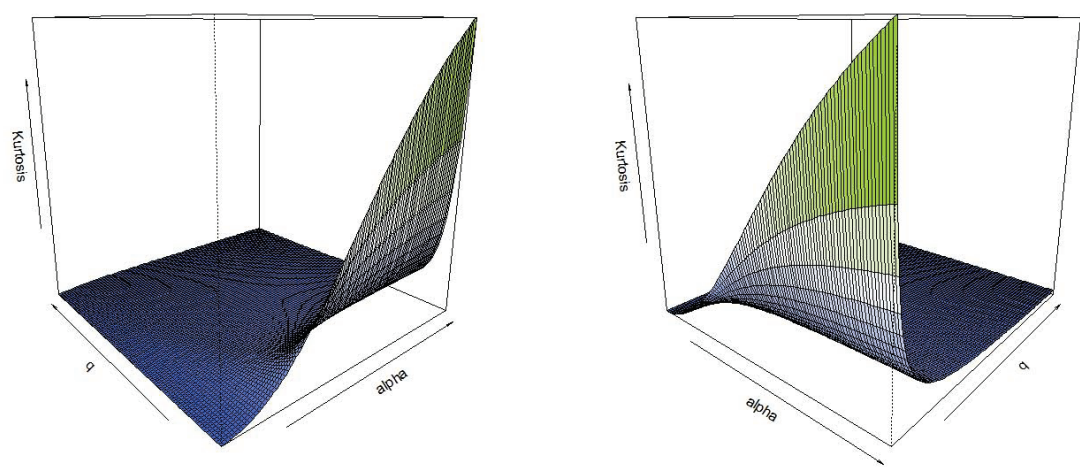

Figure 4. Kurtosis of GCR distribution.

To make it more clear, the followings are noteworthy:

(i) Recalling (2), the cdf of the generalized BS distribution is

$$
F_{\mathrm{GCR}}^{g}(t ; \alpha, \beta, 1 / 2)=1-G\left(\frac{1}{\alpha}\left(\sqrt{\frac{\beta}{t}}-\sqrt{\frac{t}{\beta}}\right)\right) .
$$

Then the inverse function of $F_{\mathrm{GCR}}^{g}(t ; \alpha, \beta, 1 / 2)$ is

$$
F_{\mathrm{GCR}}^{g-1}(x ; \alpha, \beta, 1 / 2)=\frac{\beta}{4}\left[-\alpha G^{-1}(1-x)+\sqrt{\alpha^{2}\left(G^{-1}(1-x)\right)^{2}+4}\right]^{2} .
$$

Thus, the generalized $\mathrm{BS}$ random variable $Y \sim \operatorname{GCR}(\alpha, \beta, 1 / 2 ; g)$ can be expressed as

$$
Y=\frac{\beta}{4}\left(-\alpha X+\sqrt{\alpha^{2} X^{2}+4}\right)^{2}, \text { in distribution, }
$$

where $X=G^{-1}(1-U)$ is a random variable following the baseline distribution $G$ and $U \sim \operatorname{Uniform}[0,1]$.

(ii) The ratio $f_{\mathrm{GCR}}^{g}(Y ; \alpha, \beta, p) / f_{\mathrm{GCR}}^{g}(Y ; \alpha, \beta, 1 / 2)$ is less than or equal to a positive constant depending on the value of $p:$

$$
\frac{f_{\mathrm{GCR}}^{g}(Y ; \alpha, \beta, p)}{f_{\mathrm{GCR}}^{g}\left(Y ; \alpha, \beta, \frac{1}{2}\right)} \leq c:=\max \{2 p, 2(1-p)\} .
$$

Therefore the probability of acceptance is

$$
\begin{aligned}
\operatorname{Pr}\left[V \leq \frac{f_{\mathrm{GCR}}^{g}(Y ; \alpha, \beta, p)}{c f_{\mathrm{GCR}}^{g}\left(Y ; \alpha, \beta, \frac{1}{2}\right)}\right] & =\int_{0}^{\infty} \frac{f_{\mathrm{GCR}}^{g}(y ; \alpha, \beta, p)}{c f_{\mathrm{GCR}}^{g}\left(y ; \alpha, \beta, \frac{1}{2}\right)} f_{\mathrm{GCR}}^{g}\left(y ; \alpha, \beta, \frac{1}{2}\right) d y \\
& =\frac{1}{c} \geq \frac{1}{2} .
\end{aligned}
$$

\section{Tail Properties}

In this section, we first discuss the tail properties of GCR distribution in the context of extreme value theory. The results can be viewed as an extension of Ferreira et al. (2012) in which the tail properties of generalized Birnbaum-Saunders distribution have been investigated. Then we introduce some concrete examples of heavy-tailed crack distribution families for the purpose of fitting some simulated and real catastrophic loss data sets. All mathematical proofs are given in the appendix.

\subsection{Tail Behavior of GCR Distribution}

The following Theorems 1-3 highlight the relationships between the tail of baseline distribution and that of the resulting GCR distribution in the context of classical extreme value theory and regular variation (e.g., Bingham et al., 1987 and Embrechts et al., 1997). 
Theorem 1. For a positive constant $\xi$, assume that the right tail of the baseline distribution function $G(x)$ is regularly varying with index $-\xi$ at $x=\infty$, then the right tail of GCR distribution with the baseline density function $g(x)$ is regularly varying with index $-\frac{1}{2} \xi$ at $x=\infty$. Specifically, if

$$
\lim _{x \rightarrow \infty} \frac{\bar{G}(c x)}{\bar{G}(x)}=c^{-\xi}, \quad \xi>0, \quad c>0
$$

then

$$
\lim _{x \rightarrow \infty} \frac{\bar{F}_{G C R}^{g}(c x)}{\bar{F}_{G C R}^{g}(x)}=c^{-\frac{1}{2} \xi}, \quad \xi>0, \quad c>0,
$$

where $c$ is a positive constant.

From extreme value theory, the tail behavior of a distribution is often characterized in terms of the limiting distribution of (standardized) block maxima. It turns out that there are only three asymptotic limits; Fréchet, Weibull and Gumbel extreme value distributions. In particular, a distribution $F$ belongs to the maximum domain (MDA) of the Fréchet distribution with tail index $\xi$, denoted by $F \in \operatorname{MDA}\left(\Phi_{\xi}\right)$, if and only if the right tail of the distribution is regularly varying with the index $-\xi$ at $\infty$, denoted by $\bar{F} \in \mathcal{R}_{-\xi}$. That is, $F \in \operatorname{MDA}\left(\Phi_{\xi}\right) \Leftrightarrow \bar{F} \in \mathcal{R}_{-\xi}$. Therefore, by Theorem 1 , if $G \in \operatorname{MDA}\left(\Phi_{\xi}\right)$, then $F_{\mathrm{GCR}}^{g} \in \operatorname{MDA}\left(\Phi_{\xi / 2}\right)$. In other words, if the baseline distribution $G$ belongs to the MDA of the Fréchet distribution with tail index $\xi$, then $F_{\mathrm{GCR}}^{g}$ belongs to the MDA of the Fréchet distribution with tail index $\xi / 2$.

As a special case of regular variation (when tail index $\xi=\infty$ ), the rapid variation is another way to characterize the tail of a distribution. The following theorem reveals that rapid variation of the right tail of baseline distribution can pass on to the right tail of the corresponding GCR distribution.

Theorem 2. If the right tail of the baseline distribution function $G(x)$ is rapidly varying with index $-\infty$ at $x=\infty$, then the right tail of GCR distribution with the baseline density function $g(x)$ is also rapidly varying with index $-\infty$ at $x=\infty$. Formally, for some positive real number $c$, if

$$
\lim _{x \rightarrow \infty} \frac{\bar{G}(c x)}{\bar{G}(x)}=\left\{\begin{array}{lll}
0 & \text { if } & c>1, \\
\infty & \text { if } & 0<c<1,
\end{array}\right.
$$

then

$$
\lim _{x \rightarrow \infty} \frac{\bar{F}_{G C R}^{g}(c x)}{\bar{F}_{G C R}^{g}(x)}=\left\{\begin{array}{lll}
0 & \text { if } & c>1, \\
\infty & \text { if } & 0<c<1 .
\end{array}\right.
$$

Thus, we can sum up as follows:

$$
\bar{G} \in \mathcal{R}_{-\infty} \Rightarrow \bar{F}_{G C R}^{g} \in \mathcal{R}_{-\infty}
$$

Note that the rapid variation is closely related to a characterization of the maximum domain of attraction of the Gumbel distribution, denoted by $\operatorname{MDA}(\Lambda)$; if a distribution $F \in \operatorname{MDA}(\Lambda)$ with infinite right endpoint, then the right tail of $F$ is rapidly varying; see Corollary 3.3.32 in Embrechts et al. (1997). The following theorem gives the relationship between the baseline distribution $G$ and the resulting generalized crack distribution $F_{\mathrm{GCR}}^{g}$ concerning the MDA of the Gumbel distribution.

Theorem 3. Assume the baseline distribution $G$ is a twice differentiable von Mises function on $(z, \infty)$ for some $z<\infty$ and the baseline distribution $G$ belongs to the MDA of the Gumbel distribution. Then $F_{G C R}^{g}$ also belongs to the MDA of the Gumbel distribution.

\subsection{Heavy-tailed Crack Distribution}

In the previous section, we focus on the tail relationships between the baseline and the resulting generalized crack distributions. As for the heavy-tailedness of a distribution, we use the following definition (e.g., Asmussen, 2003); a distribution $F$ is said to have a (right) heavy tail if

$$
\lim _{x \rightarrow \infty} e^{\lambda x} \bar{F}(x)=\infty,
$$

for all $\lambda>0$, which implies that the tail of $F$ is heavier than that of any exponential distribution. The class of heavytailed distributions is considerably large. In practice we often consider a class of long-tailed distributions which is a large subclass of heavy-tailed distributions. Specifically, a distribution $F$ is said to have a long (right) tail if

$$
\lim _{x \rightarrow \infty} \frac{\bar{F}(x+t)}{\bar{F}(x)}=1,
$$


for all $t>0$. In fact the class of long-tailed distributions is equivalent to the class of distributions with the limits of their hazard rate functions being zero:

$$
\lim _{x \rightarrow \infty} \frac{f(x)}{\bar{F}(x)}=0 .
$$

In other words, any distribution having zero limiting hazard rate is long-tailed distribution and thus, it is a heavy-tailed distribution.

The following theorem shows a sufficient condition for a GCR distribution to belong to a class of long-tailed distributions.

Theorem 4. If the hazard rate function of the baseline distribution $G$ converges to a finite constant, then the hazard rate function of GCR distribution with the baseline density function $g(x)$ converges to zero. That is,

$$
\lim _{x \rightarrow \infty} \frac{g(x)}{\bar{G}(x)}=c<\infty \Rightarrow \lim _{x \rightarrow \infty} \frac{f_{G C R}^{g}(x)}{\bar{F}_{G C R}^{g}(x)}=0,
$$

where $c$ is a finite constant.

\subsection{Examples of Heavy-tailed Crack Distributions}

In what follows we introduce three concrete examples of heavy-tailed crack distributions for practical applications. Specifically, the baseline densities of Student's $t$, Laplace and generalized Gaussian (GG) distribution are considered.

\section{Example 1. (Student's t crack distribution)}

The Student's $t$ crack distribution is constructed using the following Student's $t$ density with $v>0$ degrees of freedom as its baseline density function:

$$
g_{t_{v}}(x)=\frac{\Gamma\left(\frac{v+1}{2}\right)}{\sqrt{v \pi} \Gamma\left(\frac{v}{2}\right)}\left(1+\frac{x^{2}}{v}\right)^{-\left(\frac{v+1}{2}\right)} .
$$

The resulting density of the Student's $t$ crack distribution is given by

$$
f_{\mathrm{GCR}}^{t_{v}}(t)=\left(p \frac{\sqrt{\beta}}{\alpha} t^{-3 / 2}+(1-p) \frac{1}{\alpha \sqrt{\beta}} t^{-1 / 2}\right) \frac{\Gamma\left(\frac{v+1}{2}\right)}{\sqrt{v \pi} \Gamma\left(\frac{v}{2}\right)}\left[1+\frac{1}{\alpha^{2} \nu}\left(\frac{\beta}{t}+\frac{t}{\beta}-2\right)\right]^{-\left(\frac{\nu+1}{2}\right)}
$$

where $\alpha>0, \beta>0$ and $p \in[0,1]$ are the shape, scale and mixing weight parameters, respectively.

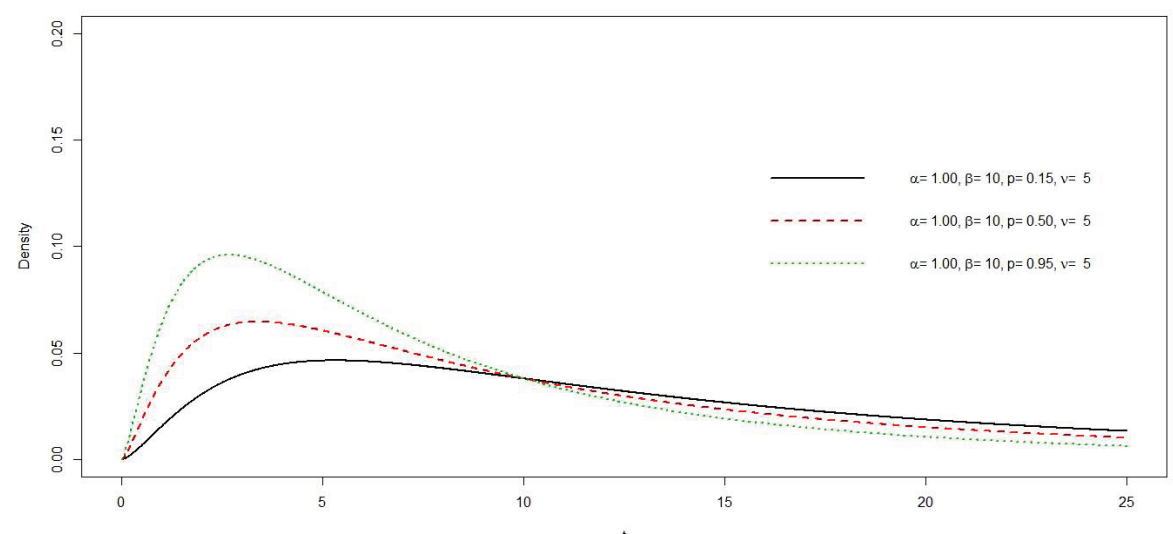

Figure 5. Density functions of Student's $t$ crack distributions.

Figure 5 illustrates the density shapes of Student's $t$ crack distributions for selected values of parameters. It can be seen that the right tail of the density function become thicker as the mixture weight $p$ gets smaller with the other parameters being fixed. Notice that

$$
f_{\mathrm{GCR}}^{g}(t ; \alpha, \beta, p)=\left[p+(1-p) \frac{t}{\beta}\right] f_{\mathrm{IS}}^{g}(t ; \alpha, \beta),
$$

and thus, at $t=\beta$, the value of the density function does not depend on $p$. In other words, the density functions of GCR distributions with the same model parameters (other than $p$ ) intersect at a single point. 
Besides, the hazard rate function of Student's $t$ distribution converges to zero, and thus, by Theorem 4 , the hazard rate function of Student's $t$ crack distribution also converges to zero. Recall that the tail behavior of GCR distribution is essentially characterized by that of the baseline distribution $G$. For some $c>0$, by L'hopital's rule, we have

$$
\lim _{x \rightarrow \infty} \frac{\bar{G}(c x)}{\bar{G}(x)}=\lim _{x \rightarrow \infty} \frac{c g(c x)}{g(x)}=c^{-v},
$$

thus Student's $t$ distribution function belongs to MDA of the Fréchet distribution with tail index $v$ (the degrees of freedom). Thus, by Theorem 1, Student's $t$ crack distribution also belongs to the MDA of the Fréchet distribution with tail index $v / 2$. Note that MDA of the Fréchet distribution contains "very heavy-tailed distributions" (Embrechts et al., 1997) and student's $t$ crack distribution may be considered for excessively heavy-tailed data distributions.

\section{Example 2. (Laplace crack distribution)}

The Laplace crack distribution uses the following Laplace density as the baseline density function:

$$
g_{\text {Laplace }}(x)=\frac{1}{2 b} \exp \left(\frac{-|x-\mu|}{b}\right),
$$

where $b>0$ and $\mu$ are the scale and location parameters, respectively. By setting $\mu=0$ for symmetry, we obtain the following density of the Laplace crack distribution:

$$
f_{\mathrm{GCR}}^{\text {Laplace }}(t)=\left(p \frac{\sqrt{\beta}}{\alpha} t^{-3 / 2}+(1-p) \frac{1}{\alpha \sqrt{\beta}} t^{-1 / 2}\right) \frac{1}{2 b} \exp \left(-\frac{1}{\alpha b}\left|\sqrt{\frac{\beta}{t}}-\sqrt{\frac{t}{\beta}}\right|\right),
$$

where $\alpha>0, \beta>0,0 \leq p \leq 1$, and the auxiliary scale parameter $b>0$. From the density, one can see that the auxiliary scale parameter $b$ and the shape parameter $\alpha$ are combined together, which results in the issue of non-identifiability in parameter estimation. To avoid the problem, we use the standardized Laplace density function as the baseline density function by setting $b=1 / \sqrt{2}$ throughout the rest of this paper.

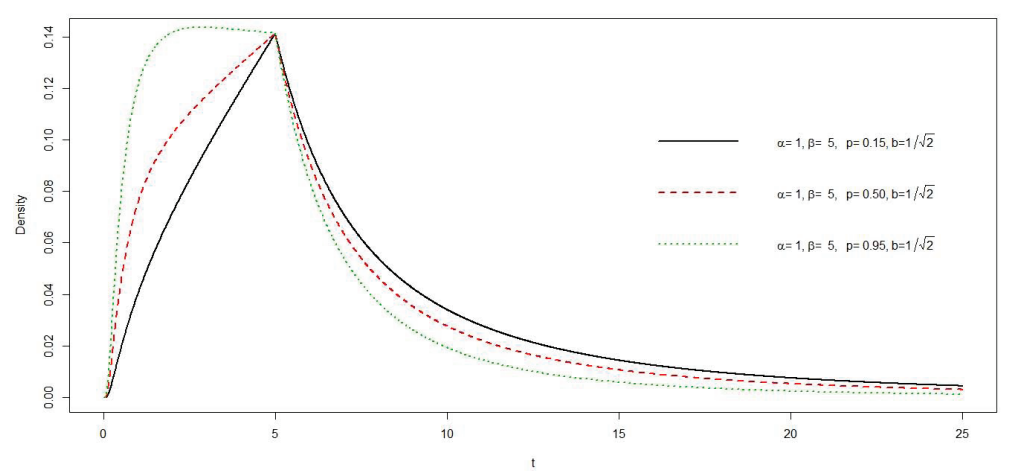

Figure 6. Density functions of Laplace crack distributions.

Figure 6 shows some interesting density shapes of Laplace crack distributions for a few sets of prescribed parameter values. Note that the density is not differentiable at $t=\beta$. Also note that the right tail of Laplace distribution is rapidly varying with index $-\infty$ at $x=\infty$. By Theorem 2 , we conclude that the Laplace crack distribution is also rapidly varying.

\section{Example 3. (Generalized Gaussian crack distribution)}

The generalized Gaussian crack distribution family is a large distribution family containing the Laplace and Gaussian crack distributions as its members. The distribution is constructed based on the following generalized Gaussian density function as its baseline density:

$$
g_{\mathrm{GG}}(x)=\frac{\theta}{2 \lambda \Gamma\left(\frac{1}{\theta}\right)} \exp \left(-\left|\frac{x-\mu}{\lambda}\right|^{\theta}\right)
$$

where the location parameter $\mu$, scale parameter $\lambda>0$ and the tail index (shape) parameter $\theta>0$. For symmetry, we set $\mu=0$ and, for the identification of parameters, we further set $\lambda=\sqrt{\Gamma(1 / \theta) / \Gamma(3 / \theta)}$. With these simplification, we obtain the following the density function of generalized Gaussian crack distribution

$$
f_{\mathrm{GCR}}^{\mathrm{GG}}(t)=\left(p \frac{\sqrt{\beta}}{\alpha} t^{-3 / 2}+(1-p) \frac{1}{\alpha \sqrt{\beta}} t^{-1 / 2}\right) \frac{\theta}{2 \lambda \Gamma\left(\frac{1}{\theta}\right)} \exp \left(-\left|\frac{1}{\alpha \lambda}\left(\sqrt{\frac{\beta}{t}}-\sqrt{\frac{t}{\beta}}\right)\right|^{\theta}\right)
$$




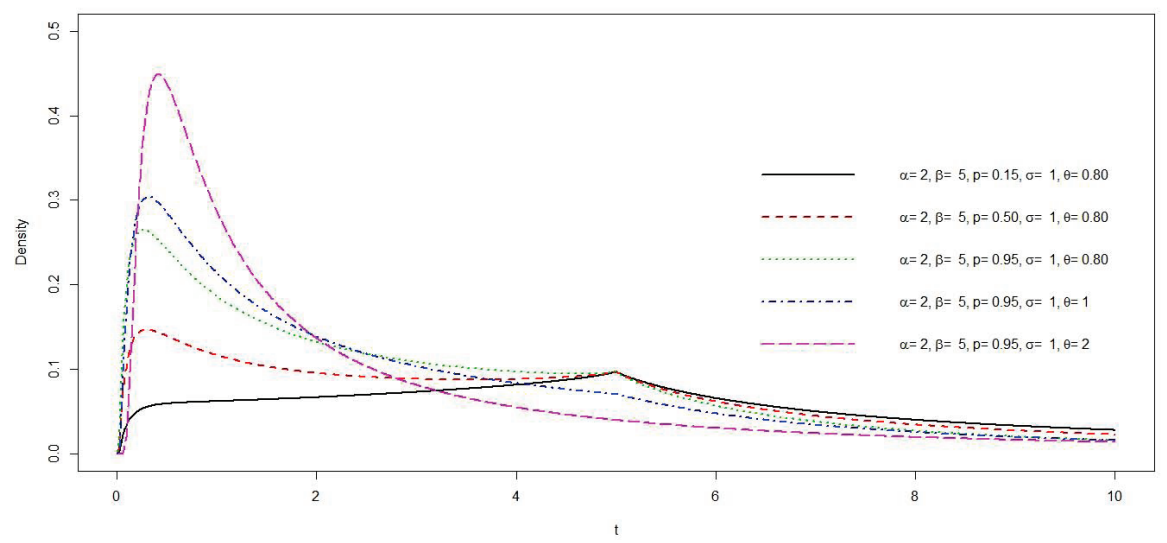

Figure 7. Density functions of generalized Gaussian crack distributions.

where $\alpha>0, \beta>0, p \in[0,1]$, and $\theta>0$.

Figure 7 illustrates various density shapes of generalized Gaussian crack distribution for a few prescribed sets of parameters. One can see from the figure that the generalized Gaussian crack distribution has a good deal of flexibility to model both thin- and heavy-tailed data. More formally, the generalized Gaussian crack distribution with shape parameter $\theta<2$ is heavy-tailed. This can be easily verified by using the following asymptotic relation:

$$
\lim _{x \rightarrow \infty} \frac{\int_{x}^{\infty} e^{-(t / \lambda)^{\theta}} d t}{x^{1-\theta} e^{-(x / \lambda)^{\theta}}}=\frac{\lambda^{\theta}}{\theta} .
$$

Further, a direct application of (3) gives that the hazard rate function of generalized Gaussian distribution with $\theta \leq$ 1 converges to a constant. By Theorem 4, we conclude that the hazard rate function of generalized Gaussian crack distribution with $\theta \leq 1$ converges to zero and thus, the generalized Gaussian crack distribution is a long-tailed distribution.

In addition, by L'hopital's rule, we can show that the right tail of generalized Gaussian distribution is rapidly varying with index $-\infty$ at $x=\infty$ :

$$
\lim _{x \rightarrow \infty} \frac{\bar{G}(c x)}{\bar{G}(x)}=\lim _{x \rightarrow \infty} \frac{c g(c x)}{g(x)}=\lim _{x \rightarrow \infty} c e^{\left(1-c^{\theta}\right)(x / \lambda)^{\theta}}=\left\{\begin{array}{lll}
0 & \text { if } & c>1, \\
\infty & \text { if } & 0<c<1 .
\end{array}\right.
$$

Then, by Theorem 2, the generalized Gaussian crack distribution is also rapidly varying. Furthermore, it can be shown that the generalized Gaussian distribution is a von Mises function belonging to the MDA of the Gumbel distribution. By Theorem 3, the generalized Gaussian crack distribution also belongs to the MDA of the Gumbel distribution. Note that distributions with various tails, ranging from moderately heavy (e.g., lognormal distribution) to light (e.g., the Gaussian distribution), are contained in $\operatorname{MDA}(\Lambda)$; see Embrechts et al. (1997).

In the following we show that the generalized Gaussian crack distribution with $\theta \leq 1$ belongs to the class of subexponential distributions, denoted by $\mathcal{S}$. As an important subclass of long-tailed distribution class, the subexponential class is defined as follows: a distribution $F$ belongs to the class $\mathcal{S}$ if the relation

$$
\lim _{x \rightarrow \infty} \frac{\overline{F^{* n}}(x)}{\bar{F}(x)}=n
$$

holds for some (or, equivalently, for all) $n=2,3, \ldots$, where $\overline{F^{* n}}$ denotes the $n$-fold convolution of $F$. By Proposition A3.16 (a) in Embrechts et al. (1997), a distribution $F$ belongs to the class of subexponential distributions, denoted by $F \in \mathcal{S}$, if and only if

$$
\lim _{t \rightarrow \infty} \int_{0}^{t} e^{y q(t)} f(y) d y=1,
$$

where hazard rate $q(x)=f(x) / \bar{F}(x)$ converges to zero.

With the fact that the hazard rate function of generalized Gaussian crack distribution with $\theta \leq 1$ is eventually decreasing to zero, it remains to show that Eq. (4) holds. From Corollary 3.3.32 (Existence of moments) and Remark 7 of Theorem A3.12 (Properties of functions of rapid variation) in Embrechts et al. (1997), suppose the right tail of a distribution $F$ 
is rapidly varying, then all power moments of $F$ are finite. Thus, all power moments of the generalized Gaussian crack distribution are finite. Then, by the series expansion, we have

$$
\begin{aligned}
\lim _{t \rightarrow \infty} \int_{0}^{t} e^{y q(t)} f(y) d y & =\lim _{t \rightarrow \infty} \int_{0}^{t}\left(1+y q(t)+\frac{[y q(t)]^{2}}{2 !}+\cdots\right) f(y) d y \\
& =\lim _{t \rightarrow \infty} F(t)+\lim _{t \rightarrow \infty} q(t) \mathrm{E}[T]+\lim _{t \rightarrow \infty} \frac{[q(t)]^{2}}{2 !} \mathrm{E}\left[T^{2}\right]+\cdots \\
& =1,
\end{aligned}
$$

where $q(t)$ is the hazard rate function of the generalized Gaussian crack distribution with $\theta \leq 1, t>0, F$ and $f$ here are the generalized Gaussian crack distribution function and the generalized Gaussian crack distribution density function, respectively, and $\mathrm{E}\left[T^{n}\right]=\int_{0}^{\infty} y^{n} f(y) d y<\infty, n=1,2,3, \ldots$ is the $n$th moment of the generalized Gaussian crack distribution.

\section{Application}

In this section, we illustrate the model fitting results on both simulated and real loss data sets. For parameter estimation, we use the maximum likelihood estimation method.

\subsection{Maximum Likelihood Estimation}

Let $\left(T_{1}, \ldots, T_{n}\right)$ be a random sample of size $n$ of the heavy-tailed crack distribution with unknown parameters $\xi=$ $(\alpha, \beta, p, \boldsymbol{\theta})$, i.e., three model parameters $(\alpha, \beta, p)$ and any additional parameters $\boldsymbol{\theta}$ involved in the baseline density function $g(\cdot ; \boldsymbol{\theta})$. The maximum likelihood estimate (MLE) of $\boldsymbol{\xi}$ are the one that maximize the following log-likelihood function:

$$
\begin{aligned}
\ell(\xi)= & \log \mathcal{L}\left(\xi ; T_{1}, \ldots, T_{n}\right)=\sum_{i=1}^{n} \log f_{\mathrm{GCR}}^{g}\left(T_{i} \mid \xi\right) \\
= & -\frac{1}{2} \sum_{i=1}^{n} \log T_{i}+\sum_{i=1}^{n} \log \left(1+\left(\frac{\beta}{T_{i}}-1\right) p\right) \\
& +\sum_{i=1}^{n} \log g\left(\frac{1}{\alpha}\left(\sqrt{\frac{\beta}{T_{i}}}-\sqrt{\frac{T_{i}}{\beta}}\right)\right)-n \log \alpha-\frac{n}{2} \log \beta .
\end{aligned}
$$

Setting aside the complexity of the baseline density $g$, the likelihood function is highly nonlinear with respect to the parameters and thus, it appears infeasible to obtain the MLE analytically. A few numerical methods can be considered to obtain the solution of the optimization problem. In particular, one may use the Expectation-Maximization (EM) algorithm (Dempster et al., 1977) which reduced to a simple two-step iterative algorithm for mixture models. In particular Lee and Lin (2010) give detailed discussions about the use of the EM algorithm for Erlang mixture models. Here we also consider an alternative iterative method, the so-called profile log-likelihood method, which consists of two nested maximization steps; see Section 4.5.2 in Davison (2003) for details. The profile log-likelihood algorithm for our applications is summarized as follows:

1. Set the starting value of the mixture weight parameter $\hat{p}^{(k)}$ where $k=0$.

2. (Step 1) Given $\hat{p}^{(k)}$, maximize the first profile log-likelihood function,

$$
l_{p_{1}}\left(\alpha, \beta, \boldsymbol{\theta} ; \hat{p}^{(k)}\right):=l\left(\alpha, \beta, \hat{p}^{(k)}, \boldsymbol{\theta}\right) .
$$

Let the solution of the optimization problem $\left(\widehat{\boldsymbol{\alpha}}^{(k+1)}, \widehat{\beta}^{(k+1)}, \widehat{\boldsymbol{\theta}}^{(k+1)}\right)$ denote the $(k+1)$ th profile likelihood estimate of $(\alpha, \beta, \boldsymbol{\theta})$.

3. (Step 2) Given $\widehat{\beta}^{(k+1)}$, maximize the second profile log-likelihood function,

$$
l_{p_{2}}\left(p ; \widehat{\beta}^{(k+1)}\right):=\sum_{i=1}^{n} \log \left(1+\left(\widehat{\beta}^{(k+1)} / T_{i}-1\right) p\right) .
$$

The solution of the optimization problem, denoted by $\widehat{p}^{(k+1)}$, is the $(k+1)$ th profile likelihood estimate of $p$, and $\widehat{\boldsymbol{\xi}}^{(k+1)}=\left(\widehat{\boldsymbol{\alpha}}^{(k+1)}, \widehat{\boldsymbol{\beta}}^{(k+1)}, \widehat{p}^{(k+1)}, \widehat{\boldsymbol{\theta}}^{(k+1)}\right)$. 
4. Repeat Step 1 and Step 2 until difference $\Delta D=\left|\ell\left(\widehat{\boldsymbol{\xi}}^{(k+1)}\right)-\ell\left(\widehat{\xi}^{(k)}\right)\right| \leq \epsilon$ for some predetermined tolerance level $\epsilon>0$.

In order to assess the finite sample performance of the estimators obtained by the profile log-likelihood method, we conduct some simulation studies. The acceptance-rejection method discussed in Section 2.3.3 is used for generating samples from the heavy-tailed crack distributions such as Student's $t$ and Laplace crack distributions. For each heavytailed crack distribution with a set of prescribed parameters, we generate random samples of size $n \in\{300,500\}$ and then, the parameters are estimated based on the proposed method. This procedure is replicated 100 times. We use the built-in R function "optim" to obtain the estimates of parameters from each generated sample.

Tables 1 and 2 give the averages and standard deviations (shown in parentheses) based on 100 estimations. The results show that the profile likelihood estimates of parameters are close to the true ones with relatively small standard deviations.

Table 1. Model fitting results to data simulated from Student's $t$ crack distribution

\begin{tabular}{|c|c|c|c|c|c|c|c|c|c|c|c|}
\hline \multirow[b]{2}{*}{$\alpha$} & \multirow[b]{2}{*}{$\beta$} & \multirow[b]{2}{*}{$p$} & \multirow[b]{2}{*}{$v$} & \multicolumn{4}{|c|}{$\mathrm{n}=300$} & \multicolumn{4}{|c|}{$\mathrm{n}=500$} \\
\hline & & & & $\widehat{\alpha}$ & $\widehat{\beta}$ & $\widehat{p}$ & $\widehat{v}$ & $\widehat{\alpha}$ & $\widehat{\beta}$ & $\widehat{p}$ & $\widehat{v}$ \\
\hline$\overline{1}$ & 5 & 0.25 & 4 & $\begin{array}{l}1.02 \\
(0.08)\end{array}$ & $\begin{array}{l}5.61 \\
(1.91)\end{array}$ & $\begin{array}{l}0.32 \\
(0.28)\end{array}$ & \begin{tabular}{|l|}
4.46 \\
$(1.86)$
\end{tabular} & $\begin{array}{l}1.01 \\
(0.08)\end{array}$ & $\begin{array}{l}5.37 \\
(1.54)\end{array}$ & $\begin{array}{l}0.29 \\
(0.23)\end{array}$ & $\begin{array}{l}4.16 \\
(1.21)\end{array}$ \\
\hline 5 & 1 & 0.50 & 5 & $\begin{array}{l}5.09 \\
(0.31)\end{array}$ & $\begin{array}{l}1.01 \\
(0.13)\end{array}$ & $\begin{array}{l}0.50 \\
(0.04)\end{array}$ & $\begin{array}{l}6.22 \\
(4.23)\end{array}$ & $\begin{array}{l}5.03 \\
(0.27)\end{array}$ & $\begin{array}{l}1.00 \\
(0.10)\end{array}$ & $\begin{array}{l}0.50 \\
(0.03)\end{array}$ & $\begin{array}{l}5.65 \\
(1.89)\end{array}$ \\
\hline 2 & 10 & 0.75 & 4 & $\begin{array}{l}2.08 \\
(0.29)\end{array}$ & $\begin{array}{l}10.71 \\
(4.41)\end{array}$ & $\begin{array}{l}0.74 \\
(0.11)\end{array}$ & $\begin{array}{l}4.71 \\
(1.58)\end{array}$ & $\begin{array}{l}2.06 \\
(0.25)\end{array}$ & $\begin{array}{l}10.72 \\
(3.63)\end{array}$ & $\begin{array}{l}0.75 \\
(0.09)\end{array}$ & $\begin{array}{l}4.46 \\
(1.17)\end{array}$ \\
\hline 10 & 2 & 0.95 & 5 & $\begin{array}{l}10.14 \\
(1.68)\end{array}$ & $\begin{array}{l}2.04 \\
(0.69)\end{array}$ & $\begin{array}{l}0.95 \\
(0.02)\end{array}$ & $\begin{array}{l}6.56 \\
(4.35)\end{array}$ & $\begin{array}{l}9.91 \\
(1.14)\end{array}$ & $\begin{array}{l}1.97 \\
(0.43)\end{array}$ & $\begin{array}{l}0.95 \\
(0.01)\end{array}$ & $\begin{array}{l}5.66 \\
(1.60)\end{array}$ \\
\hline
\end{tabular}

Table 2. Model fitting results to data simulated from the Laplace crack distribution

\begin{tabular}{|c|c|c|c|c|c|c|c|c|}
\hline \multirow[b]{2}{*}{$\alpha$} & \multirow[b]{2}{*}{$\beta$} & \multirow[b]{2}{*}{$p$} & \multicolumn{3}{|c|}{$\mathrm{n}=300$} & \multicolumn{3}{|c|}{$\mathrm{n}=500$} \\
\hline & & & $\widehat{\alpha}$ & $\widehat{\beta}$ & $\widehat{p}$ & $\widehat{\alpha}$ & $\widehat{\beta}$ & $\widehat{p}$ \\
\hline \multirow[t]{2}{*}{1} & 5 & 0.25 & 0.99 & 5.08 & 0.27 & 0.99 & 5.05 & 0.27 \\
\hline & & & $(0.05)$ & $(0.37)$ & (0.13) & $(0.04)$ & $(0.30)$ & (0.10) \\
\hline \multirow[t]{2}{*}{5} & 1 & 0.50 & 4.94 & 1.02 & 0.50 & 4.97 & 1.02 & 0.50 \\
\hline & & & $(0.28)$ & $(0.17)$ & $(0.05)$ & $(0.20)$ & (0.13) & (0.04) \\
\hline \multirow[t]{2}{*}{2} & 10 & 0.75 & 2.00 & 10.35 & 0.76 & 2.00 & 10.22 & 0.76 \\
\hline & & & $(0.15)$ & (1.68) & (0.09) & $(0.10)$ & (1.19) & $(0.07)$ \\
\hline 10 & 2 & 0.95 & $\begin{array}{c}9.94 \\
(2.10)\end{array}$ & $\begin{array}{c}2.10 \\
(0.97)\end{array}$ & $\begin{array}{c}0.94 \\
(0.03)\end{array}$ & $\begin{array}{l}10.13 \\
(2.02)\end{array}$ & $\begin{array}{c}2.18 \\
(1.24)\end{array}$ & $\begin{array}{c}0.95 \\
(0.02)\end{array}$ \\
\hline
\end{tabular}

\subsection{Model Fitting to Real Data Sets}

In this part, the results of model fitting exercises to real catastrophic loss data sets from EM-DAT (The International Disaster Database) under a few heavy-tailed crack distributions are presented. Details on the database can be found at www.emdat.be.

\subsubsection{Data Description}

The first data set contains estimated losses by storms in Asian countries ranging from 1900 to 2015, denoted by Data Set I. The second data set, denoted by Data Set II, consists of estimated losses by all natural disasters in Canada and US spanning from 1900 to 2015 .

Table 3 (unit= 1 million USD.) summaries descriptive features for these two data sets. It shows that these two data sets are both heavy-tailed and highly skewed to the right.

Table 3. Descriptive statistics for two catastrophic loss data sets

\begin{tabular}{ccccccccc}
\hline Data set & n.obs. & Min. & Med. & Max. & Mean & S.D. & Skew. & Kurt. \\
\hline Data Set I & 302 & 0.01 & 87.23 & 15143.5 & 747.22 & 1836.48 & 4.28 & 21.70 \\
Data Set II & 231 & 0.10 & 400.00 & 158230.0 & 3447.71 & 13187.09 & 8.40 & 85.55 \\
\hline
\end{tabular}

For each data set, we fit the following three concrete heavy-tailed crack distributions exemplified in Section 3.2:

1. Student's t crack distribution (GCR-t); 
2. Laplace crack distribution (GCR-Laplace);

3. the generalized Gaussian crack distribution (GCR-GG).

In contrast with the heavy-tailed crack distributions, we use three well-known models for modeling extreme data as well:

1. Log-normal distribution (Lnormal);

2. Pareto type II distribution (Pareto), i.e., Lomax distribution;

3. Weibull distribution (Weibull).

\subsubsection{Results for Data Set I}

The following table summarizes the model fitting results based on Data Set I.

From Table 4, we conclude that the GCR-GG distribution fits the data best according to maximized log-likelihoods. In addition, the other two heavy-tailed crack distributions (GCR-t and GCR-Laplace) have significantly larger maximized log-likelihoods than the Lnormal distribution and the Pareto distribution.

Table 4. Maximized log-likelihood, AIC and BIC for Data Set I

\begin{tabular}{c|c|c|c}
\hline Distribution & Log-likelihood & AIC & BIC \\
\hline GCR- $t(\alpha, \beta, p, \nu)$ & -2019.87 & 4047.74 & 4062.58 \\
GCR-Laplace $(\alpha, \beta, p)$ & -2018.58 & 4043.16 & 4054.29 \\
GCR-GG $(\alpha, \beta, p, \theta)$ & -2010.97 & 4029.93 & 4044.77 \\
\hline Lnorm $(\mu, \sigma)$ & -2025.39 & 4054.78 & 4062.20 \\
Pareto $(\alpha, \lambda)$ & -2048.42 & 4100.84 & 4108.26 \\
Weibull $(\alpha, \lambda)$ & -2017.22 & 4038.44 & 4045.86 \\
\hline
\end{tabular}

Note that the heavy-tailed crack distributions have more parameters than those three well-known two-parameter models. In this situation, one may consider some other model selection criteria such as Akaike information criterion (AIC) or Bayesian information criterion (BIC). These information criteria are commonly used to evaluate model (predictive) performance by penalizing the model complexity in terms of the number of estimated parameters. Specifically, the AIC and $\mathrm{BIC}$ are defined as follows:

$$
\begin{aligned}
\mathrm{AIC} & =-2 \ell(\hat{\boldsymbol{\xi}})+2 \times k, \\
\mathrm{BIC} & =-2 \ell(\hat{\boldsymbol{\xi}})+\log (n) \times k,
\end{aligned}
$$

where $\ell(\xi)$ is the log-likelihood function, $k$ is the number of estimated parameters, and $n$ is the sample size. The selected model is the one gives the smallest value of the information criteria. From Table 4, the GCR-GG distribution has the lowest values of both information criteria, which leads us to conclude that the GCR-GG distribution is the most appropriate among the candidate models for Data Set I.

The $p-p$ (probability to probability) plot in Figure 8 is close to the straight comparison line (the $45^{\circ}$ line from $(0,0)$ to $(1,1)$ ) on the whole, which confirms a coherence between the empirical distribution and the best fitted distribution for the data set.

\subsubsection{Resutls for Data Set II}

The same procedures used in the previous sections are applied for analyzing Data Set II. Table 5 presents the maximized log-likelihoods and the values of AIC and BIC for all candidate models. From the result, the GCR-GG model is once again the best one among all the candidate models with regard to maximized log-likelihoods value and the information criteria.

Figure 9 shows that the shape of empirical distribution roughly conforms to the best fitted distribution to the extent that the plotted points approximately match the diagonal line.

\section{Concluding Remarks}

In this paper, we studied the tail properties of generalized crack distribution. Specifically, the tail relationships between the baseline distribution and the corresponding generalized crack distribution have been identified. Relying on the theoretical properties, we have introduced a few concrete examples of heavy-tailed crack distributions such as Student's $t$, Laplace and generalized Gaussian crack distributions. These heavy-tailed crack distributions have been used for model fitting 


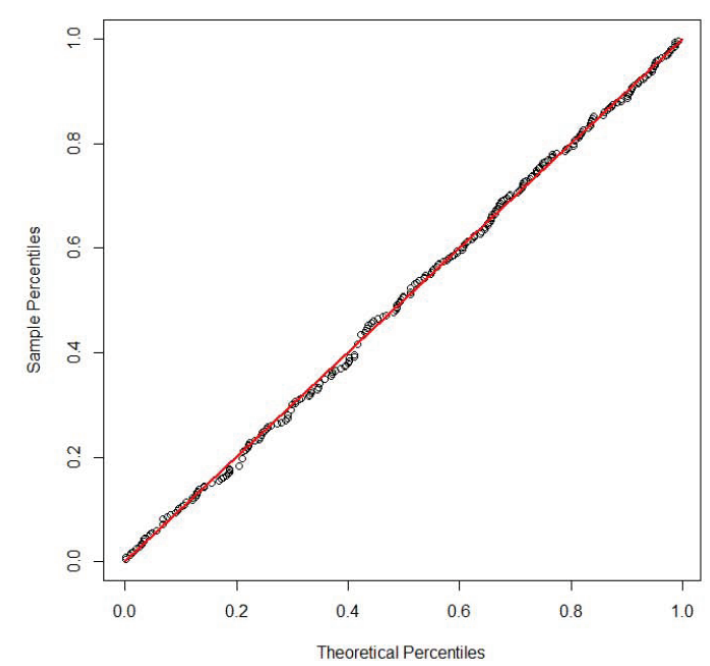

Figure 8. The $p-p$ plot of the fitted GCR-GG distribution and the empirical distribution for Data Set I.

Table 5. Maximized log-likelihood, AIC and BIC for Data Set II

\begin{tabular}{c|c|c|c}
\hline Distribution & Log-likelihood & AIC & BIC \\
\hline GCR- $t(\alpha, \beta, p, v)$ & -1859.16 & 3726.32 & 3740.09 \\
GCR-Laplace $(\alpha, \beta, p)$ & -1865.52 & 3737.04 & 3747.37 \\
GCR-GG $(\alpha, \beta, p, \theta)$ & -1855.93 & 3719.87 & 3733.64 \\
\hline Lnorm $(\mu, \sigma)$ & -1861.48 & 3726.97 & 3733.85 \\
Pareto $(\alpha, \lambda)$ & -1873.17 & 3750.34 & 3757.22 \\
Weibull $(\alpha, \lambda)$ & -1864.92 & 3733.83 & 3740.72 \\
\hline
\end{tabular}

exercises based on both simulated and real catastrophic loss data sets. The fitting results showed that the heavy-tailed crack distribution with an appropriate choice of baseline density function outperforms several parametric distributions (i.e., Log-normal, Pareto type II, Weibull distributions) commonly used in modeling highly positively skewed and heavytailed data sets.

We conclude the paper with some possible model extensions. Recall that the generalized crack distribution is a twopoint mixture of the inverse Gaussian distribution and its length-biased version. A further extension can be achieved by constructing a finite or countable mixtures of length-biased versions with various orders. Due to the extensive model flexibility, these mixture models are expected to admit further applications where the two-point mixture models are not satisfactory. Besides a multivariate extension of heavy-tailed crack distribution (e.g., Kunda et al., 2013) could also be studied in order to incorporate dependence structures among observations.

\section{Acknowledgements}

This research is supported by the Discovery Grants from the Natural Sciences and Engineering Research Council of Canada (T. Bae). 


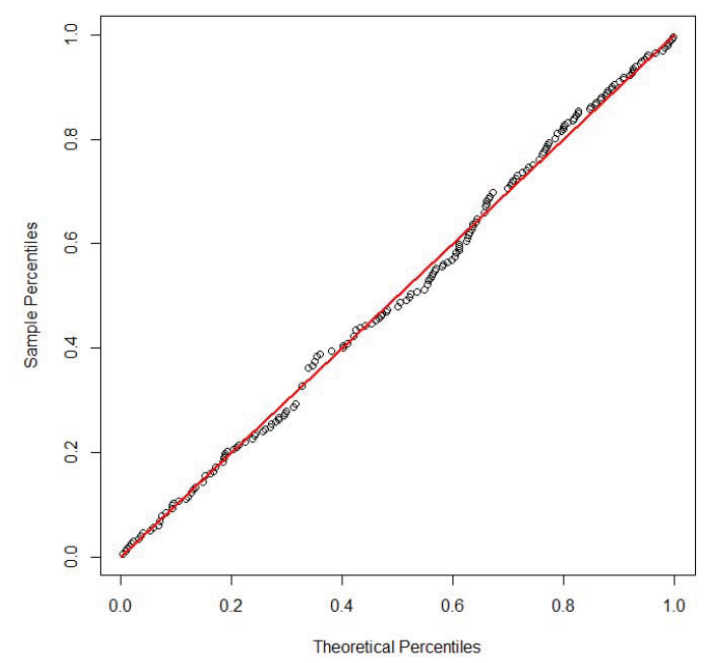

Figure 9. The $p-p$ plot of the fitted GCR-GG distribution and the empirical distribution for Data Set II.

\section{References}

Bae, T., \& Volodin, A. (2014). A generalization of three parameter crack distribution for loss severity modeling. Working paper, University of Regina.

Bingham, N. H., Goldie, C. M., \& Teugels, J. L. (1987). Regular Variation. Cambridge University Press. https://doi.org/10.1017/CBO9780511721434

Birnbaum, Z. W., \& Saunders, S. C. (1969a). A new family of life distributions. Journal of Applied Probability, (6), 319 - 327. https://doi.org/10.1017/S0021900200032848

Birnbaum, Z. W., \& Saunders, S. C. (1969b). Estimation for a family of life distributions with applications to fatigue. Journal of Applied Probability, (6), 328 - 347. https://doi.org/10.1017/S002190020003285X

Davison, A. C. (2003). Statistical models. Cambridge University Press. https://doi.org/10.1017/CBO9780511815850

Dempster, A. P., Laird, N. M., \& Rubin, D. B. (1977). Maximum likelihood from incomplete data via the EM algorithm. Journal of the Royal Statistical Society, Series B (Methodological), (39), 1 - 38.

Desmond, A. (1986). On the relationship between two fatigue-life models. IEEE Transactions on Reliability, (35), 167 169. https://doi.org/10.1109/TR.1986.4335393

Díaz-García, J. A., \& Leiva-Sánchez, V. (2005). A new family of life distributions based on the elliptically contoured distributions. Journal of Statistical Planning and Inference, (128), $445-457$.

Embrechts, P., Klüppelberg, C., \& Mikosch, T. (1997). Modelling Extremal Events for Insurance and Finance. SpringerVerlag: Berlin. https://doi.org/10.1007/978-3-642-33483-2

Ferreira, M. (2013). A study of exponential-type tails applied to Birnbaum-Saunders models. Chilean Journal of Statistics, (4), $91-101$.

Ferreira, M., Gomes, M. I., \& Leiva, V. (2012). On an extreme value version of the Birnbaum-Saunders distribution. Revstat, (10), $181-210$.

Gomes, M.I., Ferreira, M., \& Leiva, V. (2012). The extreme value Birnbaum-Saunders model, its moments and an application in biometry. Biometrical Letters, (49), 81 - 94.

Kundu, D., Balakrishnan, N., \& Jamalizadeh, A. (2013). Generalized multivariate Birnbaum-Saunders distributions and related inferential issues. Journal of Multivariate Analysis, (116), 230 - 244. https://doi.org/10.1016/j.jmva.2012.10.017

Lee, S. C. K., \& Lin, X. S. (2010). Modeling and evaluating insurance losses via mixtures of Erlang distributions. North American Actuarial Journal, (14), 107 - 130.

Leiva, V. (2015). The Birnbaum-Saunders Distribution. Academic Press. 
Leiva, V., Barros, M., Paula, G. A., \& Sanhueza. A. (2008). Generalized Birnbaum-Saunders distribution applied to air pollutant concentration. Environmetrics, (19), 235 - 249. https://doi.org/10.1002/env.861

Leiva, V., Riquelme, M., Balakrishnan, N., \& Sanhueza. A. (2008). Lifetime analysis based on the generalized BirnbaumSaunders distribution. Communications in Statistics - Theory and Methods, (21), 2079 - 2097. https://doi.org/10.1016/j.csda.2007.07.003

Leiva, V., Sanhueza, A., Kotz, S., \& Araneda, N. (2010). A unified mixture model based on the inverse Gaussian distribution. Pakistan Journal of Statistics, (26), 445 - 460.

Patil, G. P. (2002). Weighted distributions. Encyclopedia of Environmetrics (4), 2369 - 2377.

Sanhuenza, A., Leiva, V., \& Balakrishnan, N. (2008a). The generalized Birnbaum-Saunders distribution and its theory, methodology and application. Communications in Statistics - Theory and Methods, (37), 645 - 670. https://doi.org/10.1080/03610920701541174

Sanhuenza, A., Leiva, V., \& Balakrishnan, N. (2008b). A new class of inverse Gaussian type distribution. Metrika, (68). $31-49$.

Volodin, I., \& Dzhungurova, O. On limit distributions emerging in the generalized Birnbaum-Saunders model. Journal of Mathematical Sciences, (99), 1348 - 1366. https://doi.org/10.1007/BF02674095

\section{APPENDIX}

Proof of Theorem 1.

From the assumption: the right tail of the baseline distribution function $G(x)$ is regularly varying with index $-\xi$ at $x=\infty$, we have

$$
\lim _{x \rightarrow \infty} \frac{\bar{G}(c x)}{\bar{G}(x)}=c^{-\xi} \stackrel{\text { L'hopital's rule }}{\longrightarrow} \lim _{x \rightarrow \infty} \frac{g(c x)}{g(x)}=c^{-\xi-1}
$$

Then, by (1), we have

$$
\lim _{x \rightarrow \infty} \frac{\bar{F}_{\mathrm{GCR}}^{g}(c x)}{\bar{F}_{\mathrm{GCR}}^{g}(x)}=\lim _{x \rightarrow \infty} \frac{\bar{G}\left(\frac{1}{\alpha} \sqrt{\frac{c x}{\beta}}+o(1)\right)+(2 p-1) \int_{v_{1}(x)}^{\infty} g\left(\sqrt{s^{2}-4 / \alpha^{2}}\right) d s}{\bar{G}\left(\frac{1}{\alpha} \sqrt{\frac{x}{\beta}}+o(1)\right)+(2 p-1) \int_{v_{2}(x)}^{\infty} g\left(\sqrt{s^{2}-4 / \alpha^{2}}\right) d s},
$$

where

$$
\begin{aligned}
& v_{1}(x)=\sqrt{\left[\frac{1}{\alpha}\left(-\sqrt{\frac{c x}{\beta}}+o(1)\right)\right]^{2}+\frac{4}{\alpha^{2}}}, \\
& v_{2}(x)=\sqrt{\left[\frac{1}{\alpha}\left(-\sqrt{\frac{x}{\beta}}+o(1)\right)\right]^{2}+\frac{4}{\alpha^{2}}} .
\end{aligned}
$$

By letting $u=\frac{1}{\alpha} \sqrt{\frac{x}{\beta}}$, we obtain

$$
\lim _{x \rightarrow \infty} \frac{\bar{F}_{\mathrm{GCR}}^{g}(c x)}{\bar{F}_{\mathrm{GCR}}^{g}(x)}=\lim _{u \rightarrow \infty} \frac{A(u ; c)+B(u ; c, p)}{1+C(u ; p)}=c^{-\xi / 2},
$$

where

$$
\lim _{u \rightarrow \infty} A(u ; c)=\lim _{u \rightarrow \infty} \frac{\bar{G}(\sqrt{c} u+o(1))}{\bar{G}(u+o(1))}=(\sqrt{c})^{-\xi}=c^{-\xi / 2}
$$


by using L'hopital's rule and the condition that $g$ is symmetric at zero,

$$
\begin{aligned}
& \lim _{u \rightarrow \infty} B(u ; c, p) \\
& =\lim _{u \rightarrow \infty} \frac{(2 p-1) \int_{\sqrt{[-\sqrt{c} u+o(1)]^{2}+4 / \alpha^{2}}}^{\infty} g\left(\sqrt{s^{2}-\frac{4}{\alpha^{2}}}\right) d s}{\bar{G}(u+o(1))} \\
& =\lim _{u \rightarrow \infty} \frac{(2 p-1)\left[(-\sqrt{c}+o(1)) \frac{-\sqrt{c} u+o(1)}{\sqrt{[-\sqrt{c} u+o(1)]^{2}+4 / \alpha^{2}}} g(\sqrt{c} u+o(1))\right]}{(1+o(1)) g(u+o(1))} \\
& =(2 p-1)(-\sqrt{c}) \lim _{u \rightarrow \infty} \frac{g(\sqrt{c} u)}{g(u)} \\
& =(2 p-1)(-\sqrt{c})(\sqrt{c})^{-\xi-1} \\
& =(1-2 p) c^{-\xi / 2} ;
\end{aligned}
$$

similarly (or when $c=1$ ),

$$
\begin{aligned}
\lim _{u \rightarrow \infty} C(u ; p) & =\lim _{u \rightarrow \infty} \frac{(2 p-1) \int_{\sqrt{[-u+o(1)]^{2}+4 / \alpha^{2}}}^{\infty} g\left(\sqrt{s^{2}-\frac{4}{\alpha^{2}}}\right) d s}{\bar{G}(u+o(1))} \\
& =1-2 p .
\end{aligned}
$$

Proof of Theorem 2.

From the assumption that the right tail of the baseline distribution function $G(x)$ is rapidly varying with index $-\infty$ at $x=\infty$, we have

$$
\lim _{x \rightarrow \infty} \frac{\bar{G}(c x)}{\bar{G}(x)}=\left\{\begin{array}{lll}
0 & \text { if } & c>1, \\
\infty & \text { if } & 0<c<1 .
\end{array}\right.
$$

Then by L'hopital's rule, we also have

$$
\lim _{x \rightarrow \infty} \frac{g(c x)}{g(x)}=\left\{\begin{array}{lll}
0 & \text { if } & c>1, \\
\infty & \text { if } & 0<c<1 .
\end{array}\right.
$$

First, for $c>1, \sqrt{c}>1$, we have

$$
\lim _{x \rightarrow \infty} \frac{\bar{F}_{\mathrm{GCR}}^{g}(c x)}{\bar{F}_{\mathrm{GCR}}^{g}(x)}=\lim _{u \rightarrow \infty} \frac{A(u ; c)+B(u ; c, p)}{1+C(u ; p)}=0,
$$

where

$$
\begin{aligned}
\lim _{u \rightarrow \infty} A(u ; c) & =\lim _{u \rightarrow \infty} \frac{\bar{G}(\sqrt{c} u+o(1))}{\bar{G}(u+o(1))}=0, \\
\lim _{u \rightarrow \infty} B(u ; c, p) & =(2 p-1)(-\sqrt{c}) \lim _{u \rightarrow \infty} \frac{g(\sqrt{c} u)}{g(u)}=0, \\
\lim _{u \rightarrow \infty} C(u ; p) & =1-2 p .
\end{aligned}
$$

Second, for $0<c<1,0<\sqrt{c}<1$, we have

$$
\lim _{x \rightarrow \infty} \frac{\bar{F}_{\mathrm{GCR}}^{g}(x)}{\bar{F}_{\mathrm{GCR}}^{g}(c x)}=\lim _{u \rightarrow \infty} \frac{\widetilde{A}(u ; c)+\widetilde{B}(u ; c, p)}{1+\widetilde{C}(u ; p)}=0,
$$

where

$$
\lim _{u \rightarrow \infty} \widetilde{A}(u ; c)=\lim _{u \rightarrow \infty} \frac{\bar{G}(u+o(1))}{\bar{G}(\sqrt{c} u+o(1))}=\frac{1}{\infty}=0
$$




$$
\begin{aligned}
& \lim _{u \rightarrow \infty} \widetilde{B}(u ; c, p) \\
& =\lim _{u \rightarrow \infty} \frac{(2 p-1) \int_{\sqrt{[-u+o(1)]^{2}+4 / \alpha^{2}}}^{\infty} g\left(\sqrt{s^{2}-\frac{4}{\alpha^{2}}}\right) d s}{\bar{G}(\sqrt{c} u+o(1))} \\
& =\lim _{u \rightarrow \infty} \frac{(2 p-1)\left[(-1+o(1)) \frac{-u+o(1)}{\sqrt{[-u+o(1)]^{2}+4 / \alpha^{2}}} g(u+o(1))\right]}{(\sqrt{c}+o(1)) g(\sqrt{c} u+o(1))} \\
& =\frac{(1-2 p)}{\sqrt{c}} \lim _{u \rightarrow \infty} \frac{g(u+o(1))}{g(\sqrt{c} u+o(1))}=0 ; \\
& \lim _{u \rightarrow \infty} \widetilde{C}(u ; c, p) \\
& =\lim _{u \rightarrow \infty} \frac{(2 p-1) \int_{\sqrt{[-\sqrt{c} u+o(1)]^{2}+4 / \alpha^{2}}}^{\infty} g\left(\sqrt{s^{2}-\frac{4}{\alpha^{2}}}\right) d s}{\bar{G}(\sqrt{c} u+o(1))} \\
& =\lim _{u \rightarrow \infty} \frac{(2 p-1)\left[(-\sqrt{c}+o(1)) \frac{-\sqrt{c} u+o(1)}{\sqrt{[-\sqrt{c} u+o(1)]^{2}+4 / \alpha^{2}}} g(\sqrt{c} u+o(1))\right]}{(\sqrt{c}+o(1)) g(\sqrt{c} u+o(1))} \\
& =1-2 p \text {. }
\end{aligned}
$$

Thus, we obtain

$$
\lim _{x \rightarrow \infty} \frac{\bar{F}_{\mathrm{GCR}}^{g}(c x)}{\bar{F}_{\mathrm{GCR}}^{g}(x)}=\infty, \quad 0<c<1 .
$$

Proof of Theorem 3 .

From Example 3.3.23 and Proposition 3.3.25 in Embrechts et al. (1997), twice differentiable baseline distribution $G(x)$ is a von Mises function belonging to the MDA of the Gumbel distribution if and only if

$$
\lim _{x \rightarrow \infty} \frac{\bar{G}(x) G^{\prime \prime}(x)}{g^{2}(x)}=-1 .
$$

Further, it can be proved that

$$
\lim _{x \rightarrow \infty} \frac{\bar{F}_{\mathrm{GCR}}^{g}(x ; \alpha, \beta, 1 / 2) F_{\mathrm{GCR}}^{g \prime \prime}(x ; \alpha, \beta, 1 / 2)}{\left[f_{\mathrm{GCR}}^{g}(x ; \alpha, \beta, 1 / 2)\right]^{2}}=-1,
$$

which indicates that $F_{\mathrm{GCR}}^{g}(x) \in \operatorname{MDA}(\Lambda)$ when mixture weight parameter $p=\frac{1}{2}$.

However, when $p \neq \frac{1}{2}$, we prove that

$$
\lim _{x \rightarrow \infty} \frac{\bar{F}_{\mathrm{GCR}}^{g}(x) F_{\mathrm{GCR}}^{g \prime \prime}(x)}{\left[f_{\mathrm{GCR}}^{g}(x)\right]^{2}}=\lim _{x \rightarrow \infty} \frac{\bar{G}(x) G^{\prime \prime}(x)}{2(p-1) g^{2}(x)}=-\frac{1}{2(p-1)} \neq-1 .
$$

Therefore, when $p \neq \frac{1}{2}, F_{\mathrm{GCR}}^{g}(x)$ is not a von Mises function.

From extreme value theory, the MDA of the Gumbel distribution consists of von Mises distribution functions and their tail-equivalent distributions. By Proposition 3.3.28 and Definition 3.3.3 in Embrechts et al. (1997), it can be proved that two distributions $F_{\mathrm{GCR}}^{g}(x ; \alpha, \beta, p=1 / 2)$ and $F_{\mathrm{GCR}}^{g}(x ; \alpha, \beta, p \neq 1 / 2)$ are tail-equivalent:

$$
\lim _{x \rightarrow \infty} \frac{F_{\mathrm{GCR}}^{g}(x ; \alpha, \beta, 1 / 2)}{F_{\mathrm{GCR}}^{g}(x ; \alpha, \beta, p)}=\frac{1}{2(1-p)} .
$$

Then $F_{\mathrm{GCR}}^{g}(x ; \alpha, \beta, p \neq 1 / 2)$ belongs to the MDA of the Gumbel distribution as a tail-equivalent distribution of a von Mises function $F_{\mathrm{GCR}}^{g}(x ; \alpha, \beta, p=1 / 2)$; see Section 3.3.3 in Embrechts et al. (1997) for details of the maximum domain of attraction of the Gumbel distribution. 
Proof of Theorem 4.

From the assumption that the hazard rate function of the baseline distribution $G$ converges to a finite constant, we have

$$
\lim _{x \rightarrow \infty} \frac{g(x)}{\bar{G}(x)}=c<\infty .
$$

By letting $u=\frac{1}{\alpha} \sqrt{\frac{x}{\beta}}$ and using the symmetry of $g$, we obtain

$$
\begin{aligned}
& \lim _{x \rightarrow \infty} \frac{f_{\mathrm{GCR}}^{g}(x)}{\bar{F}_{\mathrm{GCR}}^{g}(x)} \\
& =\lim _{x \rightarrow \infty} \frac{\left[p \frac{\sqrt{\beta}}{\alpha} x^{-3 / 2}+(1-p) \frac{1}{\alpha \sqrt{\beta}} x^{-1 / 2}\right] g\left(\frac{1}{\alpha}\left(\sqrt{\frac{\beta}{x}}-\sqrt{\frac{x}{\beta}}\right)\right)}{G\left(\frac{1}{\alpha}\left(\sqrt{\frac{\beta}{x}}-\sqrt{\frac{x}{\beta}}\right)\right)+(2 p-1) \int_{v(x)}^{\infty} g\left(\sqrt{x^{2}-\frac{4}{\alpha^{2}}}\right) d x} \\
& =\lim _{u \rightarrow \infty} \frac{o(1) g(o(1)-u)}{G(o(1)-u)+o(1)} \\
& =\lim _{u \rightarrow \infty} \frac{g(u) o(1)}{\bar{G}(u)}=0,
\end{aligned}
$$

where

$$
v(x)=\sqrt{\left[\frac{1}{\alpha}\left(\sqrt{\frac{\beta}{x}}-\sqrt{\frac{x}{\beta}}\right)\right]^{2}+\frac{4}{\alpha^{2}}} .
$$

\section{Copyrights}

Copyright for this article is retained by the author(s), with first publication rights granted to the journal.

This is an open-access article distributed under the terms and conditions of the Creative Commons Attribution license (http://creativecommons.org/licenses/by/4.0/). 